\title{
On the Behavior of Positive Solutions of a Difference Equations System

$$
x_{n+1}=\left\{y_{n-2}+y_{n-3}\right\} / x_{n}, y_{n+1}=\left\{x_{n-2}+x_{n-3}\right\} / y_{n}^{*}
$$

\section{Decun Zhang, Wenqiang $\mathrm{Ji}^{\#}$, Liying Wang, Xiaobao Li}

Institute of Systems Science and Mathematics,

Naval Aeronautical and Astronautical University Yantai, Yantai, China

Email: \#wqyikeshu@163.com

Received March 5, 2013; revised April 5, 2013; accepted April 12, 2013

Copyright (C) 2013 Decun Zhang et al. This is an open access article distributed under the Creative Commons Attribution License, which permits unrestricted use, distribution, and reproduction in any medium, provided the original work is properly cited.

\section{ABSTRACT}

Motivated by an open problem in the literature "Dynamics of Second Order Rational Difference Equations with Open Problems and Conjecture", we introduce a difference equation system:

$x_{n+1}=\frac{y_{n-2}+y_{n-3}}{x_{n}}, y_{n+1}=\frac{x_{n-2}+x_{n-3}}{y_{n}}, \quad n=0,1, \cdots *$ where $x_{i}, y_{i} \in(0, \infty), i=-3,-2,-1,0$. We try to find out some conditions such that the solution of system converges to periodic solution. This model can be applied to the two species competition and population biology.

Keywords: Difference Equation; System; Monotonicity; Periodicity; Oscillatory

\section{Introduction}

In the monograph of Dynamics of Second Order Difference Equation [1], M. R. S. Kulenović and G. Ladas gave an open problem (see [1], p. 199) as following:

Open problem 11.4.8:

Determine whether every positive solutions of the following equation converges to a periodic solution of the corresponding equation:

$$
x_{n+1}=\frac{x_{n-2}+x_{n-3}}{x_{n}}, \quad n=0,1, \cdots .
$$

Motivated by the Open Problem, we introduce the difference equation system:

$$
x_{n+1}=\frac{y_{n-2}+y_{n-3}}{x_{n}}, \quad y_{n+1}=\frac{x_{n-2}+x_{n-3}}{y_{n}}, \quad n=0,1, \cdots .
$$

where the initial points $x_{i}, y_{i} \in(0, \infty), i=\{-3,-2,-1,0\}$

Recently, there has been great interest in studying difference equation systems. One of the reasons for this is the necessity for some techniques that can be used in in-

\footnotetext{
${ }^{*}$ Research supported by Distinguished Expert Foundation and Youth Science Foundation of Naval Aeronautical and Astronautical University.

${ }^{\#}$ Corresponding author.
}

vestigating equations arising in mathematical models describing real life situations in population biology, economics, probability theory, etc. There are many papers related to the difference equations system, such as [2-9].

In [2], Cinar studied the solutions of the system of difference equations:

$$
x_{n+1}=\frac{1}{y_{n}}, \quad y_{n+1}=\frac{y_{n}}{x_{n-1} y_{n-1}}, \quad n=0,1, \cdots,
$$

In [3], E. Camouzis and Papaschinnopoulos studied the global asymptotic behavior of positive solution of the system of rational difference equations:

$$
x_{n+1}=1+\frac{x_{n}}{y_{n-m}}, \quad y_{n+1}=1+\frac{y_{n}}{x_{n-m}}, \quad n=0,1, \cdots,
$$

In [4], Ahmet Yasar Ozban studied the system of rational difference equations:

$$
x_{n}=\frac{a}{y_{n-3}}, \quad y_{n}=\frac{b y_{n-3}}{x_{n-q} y_{n-q}}, \quad n=0,1, \cdots,
$$

In [5], Abdullah Selcuk Kurbanli et al. studied the behavior of positive solutions of the system of rational difference equations: 


$$
x_{n}=\frac{x_{n-1}}{y_{n} x_{n-1}+1}, \quad y_{n}=\frac{y_{n-1}}{x_{n} y_{n-1}+1}, \quad n=0,1, \cdots .
$$

In this paper, we try to find out some conditions such that the solution of system (2) converges to periodic solution. At the same time, we can get the oscillatory of system (2).

Before giving some results of the system (2), we need some definitions as follows [6]:

Definition 1.1 A pair of sequences of positive real numbers $\left\{x_{n}, y_{n}\right\}_{n=-3}^{\infty}$ that satisfies system (2) is a positive solution of system (2). If a positive solution of system (2) is a pair of positive constants $(\bar{x}, \bar{y})$, that solution is the equilibrium solution.

Definition 1.2 A "string" of consecutive terms $\left\{x_{s}, \cdots, x_{m}\right\}$ (resp. $\left.\left\{y_{s}, \cdots, y_{m}\right\}\right),(s \geq-3, m \leq \infty)$ is said to be a positive semicycle if $x_{i} \geq \bar{x}$ (resp. $y_{i} \geq \bar{y}$ ), $\{i \in s, \cdots, m\}, \quad x_{s-1}<\bar{x}$ (resp. $y_{s-1}<\bar{y}$ ), and $x_{m+1}<\bar{x}$ (resp. $y_{m+1}<\bar{y}$ ). Otherwise, that is said to be a negative semicycle.

A "string" of consecutive terms $\left\{\left(x_{s}, y_{s}\right), \cdots,\left(x_{m}, y_{m}\right)\right\}$ is said to be a positive(resp.negative) semicycle if $\left\{x_{s}, \cdots, x_{m}\right\},\left\{y_{s}, \cdots, y_{m}\right\}$ are positive (resp.negative) semicycle.

A solution $\left\{x_{n}\right\}$ (resp. $\left\{y_{n}\right\}$ ) oscillates about $\bar{x}$ (resp. $\bar{y}$ ) if for every $i \in N$, there exist $s, m \in N$, $s \leq i, \quad m \geq i$, such that $\left(x_{s}-\bar{x}\right)\left(x_{m}-\bar{x}\right)<0 \quad$ (resp. $\left.\left(y_{s}-c\right)\left(y_{m}-c\right)<0\right)$. We say that a solution

$\left\{x_{n}, y_{n}\right\}_{n=-3}^{\infty}$ of system oscillates about $(\bar{x}, \bar{y})$ if $\left\{x_{n}\right\}$ oscillates about $\bar{x}$ or $\left\{y_{n}\right\}$ oscillates about $\bar{y}$.

\section{Some Lemmas}

Lemma 2.1 The system (2) has a unique positive equilibrium $\bar{x}=\bar{y}=2$.

The proof of lemma 2.1 is very easy, so we omit it.

Lemma 2.2 If $p>0, q>0,(p q)^{2}>4(p+q)$, Then ever positive solution of system (2) with prime period two takes the forms

$$
\begin{aligned}
& \left(p, \frac{p q+\sqrt{(p q)^{2}-4(p+q)}}{2}\right), \\
& \left(q, \frac{p q-\sqrt{(p q)^{2}-4(p+q)}}{2}\right), \cdots,
\end{aligned}
$$

or

$$
\begin{aligned}
& \left(p, \frac{p q-\sqrt{(p q)^{2}-4(p+q)}}{2}\right), \\
& \left(q, \frac{p q+\sqrt{(p q)^{2}-4(p+q)}}{2}\right), \cdots,
\end{aligned}
$$

is a period-two solution of system (2).

Proof: Let $(p, m),(q, r),(p, m),(q, r), \cdots$, be a period-two solution of system (2).

Then, by system (2) we get

$$
\left\{\begin{array}{l}
p=\frac{m+r}{q} \\
m=\frac{q+p}{r}
\end{array}\right.
$$

We can see that (7) can be changed to

$$
p q=m+r, m r=p+q,
$$

Form (8), we can obtain

$$
\begin{aligned}
& m=\frac{p q+\sqrt{(p q)^{2}-4(p+q)}}{2} \\
& r=\frac{p q-\sqrt{(p q)^{2}-4(p+q)}}{2}
\end{aligned}
$$

or

$$
\begin{aligned}
& m=\frac{p q-\sqrt{(p q)^{2}-4(p+q)}}{2}, \\
& r=\frac{p q+\sqrt{(p q)^{2}-4(p+q)}}{2}
\end{aligned}
$$

and $(p q)^{2}>4(p+q)$

Therefore, we complete the proof.

Lemma 2.3 Assume that the initial points

$x_{i}, y_{i} \in(0, \infty), i=\{-3,-2,-1,0\}$, and $\left\{x_{n}, y_{n}\right\}_{n=-3}^{\infty}$ is $a$ positive solution of system (2). Then the following cases are true:

(a) If $0<x_{-3} \leq x_{-1}, \quad 0<x_{-2} \leq x_{0}$,

$0<x_{-1} \leq \frac{y_{-2}+y_{-3}}{x_{0}}=x_{1} ; \quad 0<y_{-3} \leq y_{-1}, \quad 0<y_{-2} \leq y_{0}$,

$0<y_{-1} \leq \frac{x_{-2}+x_{-3}}{y_{0}}=y_{1}$, then $\left\{x_{2 n}, y_{2 n}\right\}_{n=-1}^{\infty}$ and

$\left\{x_{2 n+1}, y_{2 n+1}\right\}_{n=-2}^{\infty}$ are both increasing.

(b) If $x_{-3} \geq x_{-1}>0, x_{-2} \geq x_{0}>0$,

$x_{-1} \geq \frac{y_{-2}+y_{-3}}{x_{0}}=x_{1}>0 ; \quad y_{-3} \geq y_{-1}>0, \quad y_{-2} \geq y_{0}>0$,

$y_{-1} \geq \frac{x_{-2}+x_{-3}}{y_{0}}=y_{1}>0$, then $\left\{x_{2 n}, y_{2 n}\right\}_{n=-1}^{\infty}$ and

$\left\{x_{2 n+1}, y_{2 n+1}\right\}_{n=-2}^{\infty}$ are both decreasing.

Proof: (a) By system (2), we can get

$$
x_{n+1}=\frac{y_{n-2}+y_{n-3}}{x_{n}}=\frac{y_{n-2}+y_{n-3}}{\frac{y_{n-3}+y_{n-4}}{x_{n-1}}}
$$




$$
y_{n+1}=\frac{x_{n-2}+x_{n-3}}{y_{n}}=\frac{x_{n-2}+x_{n-3}}{\frac{x_{n-3}+x_{n-4}}{y_{n-1}}}
$$

i.e.

$$
\frac{x_{n+1}}{x_{n-1}}=\frac{y_{n-2}+y_{n-3}}{y_{n-3}+y_{n-4}}, \quad \frac{y_{n+1}}{y_{n-1}}=\frac{x_{n-2}+x_{n-3}}{x_{n-3}+x_{n-4}}
$$

where $n \geq 1$.

By condition $x_{-3} \leq x_{-1}$ and (9), we get:

$$
y_{0} \leq y_{2}, x_{3} \leq x_{5}, y_{6} \leq y_{8}, x_{9} \leq x_{11}, \cdots
$$

By condition $x_{-2} \leq x_{0}$, and (9), we get:

$$
y_{1} \leq y_{3}, x_{4} \leq x_{6}, y_{7} \leq y_{9}, x_{10} \leq x_{12}, \cdots
$$

By condition $0<x_{-1} \leq \frac{y_{-2}+y_{-3}}{x_{0}}=x_{1}$, and (9), we get:

$$
y_{2} \leq y_{4}, x_{5} \leq x_{7}, y_{8} \leq y_{10}, x_{11} \leq x_{13}, \cdots
$$

Equally, we can get:

$$
\begin{gathered}
x_{0} \leq x_{2}, y_{3} \leq y_{5}, x_{6} \leq x_{8}, y_{9} \leq y_{11}, \cdots \\
x_{1} \leq x_{3}, y_{4} \leq y_{6}, x_{7} \leq x_{9}, y_{10} \leq y_{12}, \cdots \\
x_{2} \leq x_{4}, y_{5} \leq y_{7}, x_{8} \leq x_{10}, y_{11} \leq y_{13}, \cdots
\end{gathered}
$$

Hence, by induction and (10)-(15), we proof that $\left\{x_{2 n}, y_{2 n}\right\}_{n=-1}^{\infty}$ and $\left\{x_{2 n+1}, y_{2 n+1}\right\}_{n=-2}^{\infty}$ are both increasing.

Using the same method, we can prove that case (b) holds.

Therefore, we complete the proof.

\section{Lemma 2.4 Assume that}

$x_{i}, y_{i} \in(2, \infty), i=\{-3,-2,-1, \cdots\}$. Then there does not exist a positive solution $\left\{x_{n}, y_{n}\right\}_{n=-3}^{\infty}$ of system (2) such that $\left\{x_{2 n}, y_{2 n}\right\}_{n=-1}^{\infty}$ and $\left\{x_{2 n+1}, y_{2 n+1}\right\}_{n=-2}^{\infty}$ are both increasing or both decreasing.

Proof: By Equation (9), we can get $\left\{x_{2 n}, y_{2 n}\right\}$ and $\left\{x_{2 n+1}, y_{2 n+1}\right\}$ have the same monotonous.

Firstly, we proof that there does not exist positive solution $\left\{x_{n}, y_{n}\right\} \in(2, \infty) \times(2, \infty)$ such that $\left\{x_{2 n}, y_{2 n}\right\}_{n=-1}^{\infty}$ and $\left\{x_{2 n+1}, y_{2 n+1}\right\}_{n=-2}^{\infty}$ are both increasing.

Assume, for the sake of contradiction, that we have the following results:

(i) $\left\{x_{2 n}, y_{2 n}\right\}_{n=-1}^{\infty}$ is increasing;

(ii) $\left\{x_{2 n+1}, y_{2 n+1}\right\}_{n=-2}^{\infty}$ is also increasing.

By system (2), we obtain

$$
\begin{aligned}
x_{n+1} & =\frac{y_{n-2}+y_{n-3}}{x_{n}} \\
& <\frac{1}{2}\left(y_{n-2}+y_{n-3}\right) \leq \max \left\{y_{n-2}, y_{n-3}\right\},
\end{aligned}
$$

$$
\begin{aligned}
y_{n+1} & =\frac{x_{n-2}+x_{n-3}}{y_{n}} \\
& <\frac{1}{2}\left(x_{n-2}+x_{n-3}\right) \leq \max \left\{x_{n-2}, x_{n-3}\right\},
\end{aligned}
$$

in Equations (16) and (17), it implies that:

$$
x_{n+1} \leq \max \left\{y_{n-2}, y_{n-3}\right\} \leq \max \left\{x_{n-5}, x_{n-6}, x_{n-7}\right\},
$$

Because of $x_{n-7} \leq x_{n-5}, x_{n-8} \leq x_{n-6}$, we can get

$$
\begin{gathered}
x_{n+1} \leq \max \left\{x_{n-5}, x_{n-6}, x_{n-7}\right\} \leq \max \left\{x_{n-5}, x_{n-6}\right\}, \\
x_{n} \leq \max \left\{x_{n-6}, x_{n-7}, x_{n-8}\right\} \leq \max \left\{x_{n-5}, x_{n-6}\right\},
\end{gathered}
$$

i.e

$$
\left\{\begin{array}{l}
x_{n+1} \leq \max \left\{x_{n-5}, x_{n-6}\right\} \\
x_{n} \leq \max \left\{x_{n-5}, x_{n-6}\right\}
\end{array}\right.
$$

Also, we can get

$$
\left\{\begin{array}{l}
y_{n+1} \leq \max \left\{y_{n-5}, y_{n-6}\right\} \\
y_{n} \leq \max \left\{y_{n-5}, y_{n-6}\right\}
\end{array}\right.
$$

Because of the assumptions (i) and (ii), it is easy to see that (18) and (19) do not hold.

This is a contradiction and we proof the case of increasing does not hold.

Next, we proof there does not exist positive solution of system (2) such that $\left\{x_{2 n}, y_{2 n}\right\}_{n=-1}^{\infty}$ and $\left\{x_{2 n+1}, y_{2 n+1}\right\}_{n=-2}^{\infty}$ are both decreasing.

Assume, for the sake of contradiction, that we have the following results:

(i) $\left\{x_{2 n}, y_{2 n}\right\}_{n=-1}^{\infty}$ is decreasing;

(ii) $\left\{x_{2 n+1}, y_{2 n+1}\right\}_{n=-2}^{\infty}$ is also decreasing.

By the Limiting Theorem we know that $\left\{x_{2 n}, y_{2 n}\right\}_{n=-1}^{\infty}$ and $\left\{x_{2 n+1}, y_{2 n+1}\right\}_{n=-2}^{\infty}$ are both decreasing into a pair of constants.

We set $p=\lim _{n \rightarrow \infty} x_{2 n}, q=\lim _{n \rightarrow \infty} x_{2 n+1}, \quad m=\lim _{n \rightarrow \infty} y_{2 n}$, $r=\lim _{n \rightarrow \infty} y_{2 n+1}$, and $p>2, q>2, m>2, r>2$.

By system (2), we know that these constants satisfy the system (2), i.e.

$$
\left\{\begin{array}{l}
m r=p+q \\
p q=m+r
\end{array}\right.
$$

However, if $p>2, q>2, m>2, r>2$, Equation (20) do not holds, which is contradiction.

Hence, we complete the proof of lemma 10.

Lemma 2.5 Assume that

$x_{i}, y_{i} \in(0,2), i=\{-3,-2,-1, \cdots\}$. Then there does not exist a positive solution $\left\{x_{n}, y_{n}\right\}_{n=-3}^{\infty}$ of system (2) such 
that $\left\{x_{2 n}, y_{2 n}\right\}_{n=-1}^{\infty}$ and $\left\{x_{2 n+1}, y_{2 n+1}\right\}_{n=-2}^{\infty}$ are both decreasing or both increasing.

Proof: First, we proof there does not exist positive solution of system (2) such that $\left\{x_{2 n}, y_{2 n}\right\}_{n=-1}^{\infty}$ and $\left\{x_{2 n+1}, y_{2 n+1}\right\}_{n=-2}^{\infty}$ are both decreasing, the proof of increasing is similar, so we omit it.

Assume, for the sake of contradiction, that we have the following results:

(i) $\left\{x_{2 n}, y_{2 n}\right\}_{n=-1}^{\infty}$ is decreasing;

(ii) $\left\{x_{2 n+1}, y_{2 n+1}\right\}_{n=-2}^{\infty}$ is also decreasing.

We set $\lim _{n \rightarrow \infty} x_{2 n}=p, \quad \lim _{n \rightarrow \infty} x_{2 n+1}=q, \lim _{n \rightarrow \infty} y_{2 n}=m$, $\lim _{n \rightarrow \infty} y_{2 n+1}=r$.

By Limit Theorem,we know that $\left\{x_{2 n}, y_{2 n}\right\}_{n=-1}^{\infty}$ and $\left\{x_{2 n+1}, y_{2 n+1}\right\}_{n=-2}^{\infty}$ are both decreasing into a pair of constants.

Obviously, the limits of $\left\{x_{2 n}, y_{2 n}\right\}_{n=-1}^{\infty}$

$\left\{x_{2 n+1}, y_{2 n+1}\right\}_{n=-2}^{\infty}$ can not decrease into zero.

By system (2), we can get

$$
\left\{\begin{array}{l}
p=\frac{m+r}{q} \\
m=\frac{q+p}{r}
\end{array}\right.
$$

where $p, q, m, r \in(0,2)$, which can be changed to

$$
\left\{\begin{array}{l}
p q=m+r \\
m r=p+q
\end{array}\right.
$$

However, if $p, q, m, r \in(0,2)$, Equation (22) can not hold.

This is a contradiction and we complete the proof.

The the proof of the case of increasing is similar with the proof of the the case of decreasing, so we omit it.

In addition to the method above, we can proof the Lemma 2.5 by the method of Lemma 2.4. Here, we omit it.

\section{Main Results}

Theorem 3.1 Assume that $x_{-3} \geq x_{-1}>0, x_{-2} \geq x_{0}>0$, $x_{-1} \geq \frac{y_{-2}+y_{-3}}{x_{0}}=x_{1}>0 ; \quad y_{-3} \geq y_{-1}>0, \quad y_{-2} \geq y_{0}>0$, $y_{-1} \geq \frac{x_{-2}+x_{-3}}{y_{0}}=y_{1}>0$, and $\left\{x_{n}, y_{n}\right\}_{n=-3}^{\infty}$ is a positive solution of system (2). Then $\left\{x_{2 n}, y_{2 n}\right\}_{n=-1}^{\infty}$ and $\left\{x_{2 n+1}, y_{2 n+1}\right\}_{n=-2}^{\infty}$ are both decreasing; and $\left\{x_{n}, y_{n}\right\}_{n=-3}^{\infty}$ converges to a period-two solution as following

$$
\begin{aligned}
& \left(p_{1}, p_{2}\right),\left(p_{3}, p_{4}\right),\left(p_{1}, p_{2}\right),\left(p_{3}, p_{4}\right), \\
& \cdots,\left(p_{1}, p_{2}\right),\left(p_{3}, p_{4}\right), \cdots .
\end{aligned}
$$

where $p_{1}, p_{2}, p_{3}, p_{4}$ satisfy $p_{1} p_{3}=p_{2}+p_{4}$, $p_{2} p_{4}=p_{1}+p_{3}$.

Proof: By lemma 2.3(a), we can obtain that $\left\{x_{2 n}, y_{2 n}\right\}_{n=-1}^{\infty}$ and $\left\{x_{2 n+1}, y_{2 n+1}\right\}_{n=-2}^{\infty}$ are both decreasing.

Then by the Limit Theorem, we can get $\lim _{n \rightarrow \infty} x_{2 n}$, $\lim _{n \rightarrow \infty} y_{2 n}, \lim _{n \rightarrow \infty} x_{2 n+1}$, and $\lim _{n \rightarrow \infty} y_{2 n+1}$, all exist and are positive.

We can set

$\lim _{n \rightarrow \infty} x_{2 n}=p_{1}, \lim _{n \rightarrow \infty} y_{2 n}=p_{2}, \lim _{n \rightarrow \infty} x_{2 n+1}=p_{3}, \lim _{n \rightarrow \infty} y_{2 n+1}=p_{4}$

By lemmas 2.4 and 2.5, we know that there does not exist a positive solution $\left\{x_{n}, y_{n}\right\}_{n=-3}^{\infty} \in(0,2) \times(0,2)$ or $\left\{x_{n}, y_{n}\right\}_{n=-3}^{\infty} \in(2, \infty) \times(2, \infty)$ such that $\left\{x_{2 n}, y_{2 n}\right\}_{n=-1}^{\infty}$ and $\left\{x_{2 n+1}, y_{2 n+1}\right\}_{n=-2}^{\infty}$ are both decreasing.

Hence, there is at least one of $p_{i}$ satisfy $p_{i} \in(0,2)$ and at least one of $p_{i}$ satisfy $p_{i}>2$

By system (2), we get

$$
\left\{\begin{array}{l}
p_{3}=\frac{p_{2}+p_{4}}{p_{1}} \\
p_{4}=\frac{p_{1}+p_{3}}{p_{2}}
\end{array}\right.
$$

It is to see that $\left(p_{1}, p_{2}\right),\left(p_{3}, p_{4}\right),\left(p_{1}, p_{2}\right),\left(p_{3}, p_{4}\right), \cdots$, is a period-two solution of system (2), and $p_{1}, p_{2}, p_{3}, p_{4}$ satisfy $p_{1} p_{3}=p_{2}+p_{4}, \quad p_{2} p_{4}=p_{1}+p_{3}$.

We complete the proof.

Corollary 3.1 Suppose that $\left\{x_{n}, y_{n}\right\}_{n=-3}^{\infty}$ is a positive solution of system (2). Then the following statement is true:

If $x_{-3} \geq x_{-1}>0, \quad x_{-2} \geq x_{0}>0$,

$x_{-1} \geq \frac{y_{-2}+y_{-3}}{x_{0}}=x_{1}>0 ; \quad y_{-3} \geq y_{-1}>0, \quad y_{-2} \geq y_{0}>0$,

$y_{-1} \geq \frac{x_{-2}+x_{-3}}{y_{0}}=y_{1}>0$, the solution of system (2)

$\left\{x_{n}, y_{n}\right\}_{n=-3}^{\infty}$ eventually oscillates about equilibrium $(\bar{x}, \bar{y})$.

Theorem 3.2 Assume that $0<x_{-3} \leq x_{-1}, \quad 0<x_{-2} \leq x_{0}$, $0<x_{-1} \leq \frac{y_{-2}+y_{-3}}{x_{0}}=x_{1} ; \quad 0<y_{-3} \leq y_{-1}, \quad 0<y_{-2} \leq y_{0}$, $0<y_{-1} \leq \frac{x_{-2}+x_{-3}}{y_{0}}=y_{1}$, and $\left\{x_{n}, y_{n}\right\}_{n=-3}^{\infty}$ is a positive solution of system (2). Then $\left\{x_{2 n}, y_{2 n}\right\}_{n=-1}^{\infty}$ and $\left\{x_{2 n+1}, y_{2 n+1}\right\}_{n=-2}^{\infty}$ are both increasing; and $\left\{x_{n}\right\}_{n=-3}^{\infty}$ converges to a period-two solution as following

$$
\begin{aligned}
& \left(p_{1}, p_{2}\right),\left(p_{3}, p_{4}\right),\left(p_{1}, p_{2}\right),\left(p_{3}, p_{4}\right), \\
& \cdots,\left(p_{1}, p_{2}\right),\left(p_{3}, p_{4}\right), \cdots .
\end{aligned}
$$


where $p_{1}, p_{2}, p_{3}, p_{4}$ satisfy $p_{1} p_{3}=p_{2}+p_{4}$,

$p_{2} p_{4}=p_{1}+p_{3}$.

Proof: By lemma 2.3(a), we obtain that $\left\{x_{2 n}, y_{2 n}\right\}_{n=-1}^{\infty}$ and $\left\{x_{2 n+1}, y_{2 n+1}\right\}_{n=-2}^{\infty}$ are both increasing.

We set $\lim _{n \rightarrow \infty} x_{2 n}=p_{1}, \lim _{n \rightarrow \infty} y_{2 n}=p_{2}, \lim _{n \rightarrow \infty} x_{2 n+1}=p_{3}$, $\lim _{n \rightarrow \infty} y_{2 n+1}=p_{4}$

By Equation (9), we can get

$$
\left\{\begin{array}{l}
\frac{x_{n+1}}{x_{n-1}}=\frac{y_{n-2}+y_{n-3}}{y_{n-3}+y_{n-4}}<\frac{y_{n-2}}{y_{n-4}} \\
\frac{y_{n+1}}{y_{n-1}}=\frac{x_{n-2}+x_{n-3}}{x_{n-3}+x_{n-4}}<\frac{x_{n-2}}{x_{n-4}}
\end{array}\right.
$$

which can be changed into:

$$
\begin{aligned}
& \frac{y_{n-4}}{y_{n-2}}<\frac{x_{n-1}}{x_{n+1}}<\frac{y_{n+2}}{y_{n+4}}<\frac{x_{n+5}}{x_{n+7}} \\
& \frac{x_{n-4}}{x_{n-2}}<\frac{y_{n-1}}{y_{n+1}}<\frac{x_{n+2}}{x_{n+4}}<\frac{y_{n+5}}{y_{n+7}}
\end{aligned}
$$

By the $x_{i}, y_{i} \in(0, \infty), i=\{-3,-2,-1,0\}$, we can get

$$
\begin{aligned}
& \frac{x_{-3}}{x_{-1}}<\frac{y_{0}}{y_{2}}<\frac{x_{3}}{x_{5}}<\frac{y_{6}}{y_{8}}<\frac{x_{9}}{x_{11}}<\frac{y_{12}}{y_{14}}<\frac{x_{15}}{x_{17}}<\frac{y_{18}}{y_{20}} \\
& <\frac{x_{21}}{x_{23}}<\frac{y_{24}}{y_{26}}<\frac{x_{27}}{x_{29}}<\frac{y_{30}}{y_{32}}<\cdots, \\
& \frac{x_{-2}}{x_{0}}<\frac{y_{1}}{y_{3}}<\frac{x_{4}}{x_{6}}<\frac{y_{7}}{y_{9}}<\frac{x_{10}}{x_{12}}<\frac{y_{13}}{y_{15}}<\frac{x_{16}}{x_{18}}<\frac{y_{19}}{y_{21}} \\
& <\frac{x_{22}}{x_{24}}<\frac{y_{25}}{y_{27}}<\frac{x_{28}}{x_{30}}<\frac{y_{31}}{y_{33}}<\cdots, \\
& \frac{x_{-1}}{x_{1}}<\frac{y_{2}}{y_{4}}<\frac{x_{5}}{x_{7}}<\frac{y_{8}}{y_{10}}<\frac{x_{11}}{x_{13}}<\frac{y_{14}}{y_{16}}<\frac{x_{17}}{x_{19}}<\frac{y_{20}}{y_{22}} \\
& <\frac{x_{23}}{x_{25}}<\frac{y_{26}}{y_{28}}<\frac{x_{29}}{x_{31}}<\frac{y_{32}}{y_{34}}<\cdots, \\
& \frac{y_{-3}}{y_{-1}}<\frac{x_{0}}{x_{2}}<\frac{y_{3}}{y_{5}}<\frac{x_{6}}{x_{8}}<\frac{y_{9}}{y_{11}}<\frac{x_{12}}{x_{14}}<\frac{y_{15}}{y_{17}}<\frac{x_{18}}{x_{20}} \\
& <\frac{y_{21}}{y_{23}}<\frac{x_{24}}{x_{26}}<\frac{y_{27}}{y_{29}}<\frac{x_{30}}{x_{32}}<\cdots, \\
& \frac{y_{-2}}{y_{0}}<\frac{x_{1}}{x_{3}}<\frac{y_{4}}{y_{6}}<\frac{x_{7}}{x_{9}}<\frac{y_{10}}{y_{12}}<\frac{y_{13}}{y_{15}}<\frac{x_{16}}{x_{18}}<\frac{y_{19}}{y_{21}} \\
& <\frac{x_{22}}{x_{24}}<\frac{x_{25}}{x_{27}}<\frac{y_{28}}{y_{30}}<\frac{x_{31}}{x_{33}}<\cdots, \\
& \frac{y_{-1}}{y_{1}}<\frac{x_{2}}{x_{4}}<\frac{y_{5}}{y_{7}}<\frac{x_{8}}{x_{10}}<\frac{y_{11}}{y_{13}}<\frac{x_{14}}{x_{16}}<\frac{y_{17}}{y_{19}}<\frac{x_{20}}{x_{22}} \\
& <\frac{y_{23}}{y_{25}}<\frac{x_{26}}{x_{28}}<\frac{y_{29}}{y_{31}}<\frac{x_{32}}{x_{34}}<\cdots \text {. }
\end{aligned}
$$

By induction, we can get

$$
\begin{aligned}
& \frac{x_{-3}}{x_{2 n+1}}<\frac{y_{0}}{y_{2 n+4}}<\frac{x_{3}}{x_{2 n+7}}<\frac{y_{6}}{y_{2 n+10}}<\frac{x_{9}}{x_{2 n+13}} \\
& \frac{x_{-2}}{x_{2 n+2}}<\frac{y_{1}}{y_{2 n+5}}<\frac{x_{4}}{x_{2 n+8}}<\frac{y_{7}}{y_{2 n+11}}<\frac{x_{10}}{x_{2 n+14}}
\end{aligned}
$$

From Lemma 10, we know that there at least one $p_{i}<2$. Then by Limiting Theorem, we can get at least one of the limiting of $p_{i}$ must exist. With no loss generality, we set the limit of $\left\{x_{2 n+1}\right\}$ exist,we can know $p_{3}<\infty$.

By limiting Equation (27), we can get

$$
\begin{aligned}
& \lim _{n \rightarrow \infty} \frac{x_{-3}}{x_{2 n+1}} \leq \lim _{n \rightarrow \infty} \frac{y_{0}}{y_{2 n+4}} \leq \lim _{n \rightarrow \infty} \frac{x_{3}}{x_{2 n+7}} \\
& \leq \lim _{n \rightarrow \infty} \frac{y_{6}}{y_{2 n+10}} \leq \lim _{n \rightarrow \infty} \frac{x_{9}}{x_{2 n+13}}
\end{aligned}
$$

Hence, we can get

$$
\frac{x_{-3}}{p_{3}} \leq \lim _{n \rightarrow \infty} \frac{y_{0}}{y_{2 n+4}} \leq \frac{x_{3}}{p_{3}}
$$

i.e.

$$
p_{2}<\infty
$$

Next, we try to proof $p_{1}<\infty$, and $p_{1}<\infty$.

By system (2), we get

$$
\begin{aligned}
& \left\{\begin{array}{l}
x_{2 n+2} x_{2 n+1}=y_{2 n-1}+y_{2 n-2} \\
y_{2 n+2} y_{2 n+1}=x_{2 n-1}+x_{2 n-2}
\end{array}\right. \\
& \left\{\begin{array}{l}
x_{2 n+3} x_{2 n+2}=y_{2 n}+y_{2 n-1} \\
y_{2 n+3} y_{2 n+2}=x_{2 n}+x_{2 n-1}
\end{array}\right.
\end{aligned}
$$

By (30) and (31), we can get

$$
\begin{aligned}
& \left\{\begin{array}{l}
x_{2 n+2}=\frac{y_{2 n}-y_{2 n-2}}{x_{2 n+3}-x_{2 n+1}} \\
y_{2 n+2}=\frac{x_{2 n}-x_{2 n-2}}{x_{2 n+3}-y_{2 n+1}}
\end{array}\right. \\
& \left\{\begin{array}{l}
x_{2 n+3}=\frac{y_{2 n+1}-y_{2 n-1}}{x_{2 n+4}-x_{2 n+2}} \\
y_{2 n+3}=\frac{x_{2 n+1}-x_{2 n-1}}{x_{2 n+4}-y_{2 n+2}}
\end{array}\right.
\end{aligned}
$$

which can be changed into

$$
\left\{\begin{array}{l}
x_{2 n+2}=\frac{1}{y_{2 n-1}} \frac{x_{2 n-3}-x_{2 n-5}}{x_{2 n+3}-x_{2 n+1}} \\
y_{2 n+2}=\frac{1}{x_{2 n-1}} \frac{y_{2 n-3}-y_{2 n-5}}{y_{2 n+3}-y_{2 n+1}}
\end{array}\right.
$$




$$
\left\{\begin{array}{l}
x_{2 n+3}=\frac{1}{y_{2 n}} \frac{x_{2 n-2}-x_{2 n-4}}{x_{2 n+4}-x_{2 n+2}} \\
y_{2 n+3}=\frac{1}{x_{2 n}} \frac{y_{2 n-2}-y_{2 n-4}}{y_{2 n+4}-y_{2 n+2}}
\end{array}\right.
$$

By the both side of Equation (35), we can get

$$
\lim _{n \rightarrow \infty} x_{2 n+3} y_{2 n}=\lim _{n \rightarrow \infty} \frac{x_{2 n-2}-x_{2 n-4}}{x_{2 n+4}-x_{2 n+2}}=p_{2} p_{3}
$$

Assume $\lim _{n \rightarrow \infty} x_{2 n}=+\infty$, by Stolz Theorem we obtain that

$$
\lim _{n \rightarrow \infty} \frac{x_{2 n-2}-x_{2 n-4}}{x_{2 n+4}-x_{2 n+2}}=\lim _{n \rightarrow \infty} \frac{x_{2 n-2}}{x_{2 n+4}}=p_{2} p_{3}
$$

Because $\frac{x_{2 n-2}}{x_{2 n+4}}<1$, then we can get the limit of $\lim _{n \rightarrow \infty} \frac{x_{2 n-2}}{x_{2 n+4}}=p_{2} p_{3} \leq 1$

However, there exist $p_{2}, p_{3}$ such that $p_{2} p_{3} \geq 1$, which is conduction.

Hence, the assume does not hold. We obtain $\lim _{n \rightarrow \infty} x_{2 n}=p_{1}<+\infty$.

Use the same method, we can also get $\lim _{n \rightarrow \infty} y_{2 n+1}=p_{4}<+\infty$.

By system (2), we get

$$
\left\{\begin{array}{l}
p_{3}=\frac{p_{2}+p_{4}}{p_{1}} \\
p_{4}=\frac{p_{1}+p_{3}}{p_{2}}
\end{array}\right.
$$

It is to see that $\left(p_{1}, p_{2}\right),\left(p_{3}, p_{4}\right),\left(p_{1}, p_{2}\right),\left(p_{3}, p_{4}\right), \cdots$, is a period-two solution of system (2), and $p_{1}, p_{2}, p_{3}, p_{4}$ satisfy $p_{1} p_{3}=p_{2}+p_{4}, p_{2} p_{4}=p_{1}+p_{3}$.

Therefore, we complete the proof.

Corollary 3.2 Suppose that $\left\{x_{n}, y_{n}\right\}_{n=-3}^{\infty}$ is a positive solution of system (2). Then the following statement is true:

$$
\begin{aligned}
& \text { If } 0<x_{-3} \leq x_{-1}, 0<x_{-2} \leq x_{0}, 0<x_{-1} \leq \frac{y_{-2}+y_{-3}}{x_{0}}=x_{1} ; \\
& 0<y_{-3} \leq y_{-1}, \quad 0<y_{-2} \leq y_{0}, \quad 0<y_{-1} \leq \frac{x_{-2}+x_{-3}}{y_{0}}=y_{1},
\end{aligned}
$$

then the solution of system (2) oscillates about equilibrium $(\bar{x}, \bar{y})$.

Theorem 3.3 Assume that $0<x_{-3}=x_{-1}, \quad 0<x_{-2}=x_{0}$, $x_{-1}=\frac{y_{-2}+y_{-3}}{x_{0}}=x_{1} ; 0<y_{-3}=y_{-1}, \quad 0<y_{-2}=y_{0}$, $y_{-1}=\frac{x_{-2}+x_{-3}}{y_{0}}=y_{1}$, and $\left\{x_{n}, y_{n}\right\}_{n=-3}^{\infty}$ is a positive solu- tion of system (2). Then the system (2) has prime period two solutions, and $x_{2 n-1}=x_{-3}, x_{2 n}=x_{-2}, \quad y_{2 n-1}=y_{-3}$, $y_{2 n}=y_{-2}$, for $n=\{-1,0,1, \cdots\}$.

Proof: By the lemma 8, we can complete the proof. Here, we omit it.

\section{REFERENCES}

[1] M. R. S. Kulenović and G. Ladas, "Dynamics of Second Order Rational Difference Equation with Open Problems and Conjectures," CRC Press, Chapman Hall, 2002.

[2] C. Cinar, "On the Positive Solutions of the Difference Equation System $x_{n+1}=\frac{1}{y_{n}}, \quad y_{n+1}=\frac{y_{n}}{x_{n-1} y_{n-1}}, "$ Applied Mathematics and Computation, Vol. 158, 2004, pp. 303305.

[3] E. Camouzis and G. Papaschinopoulos, "Global Asymptotic Behavior of Positive Solutions on the System of Rational Difference Equations

$x_{n+1}=1+\frac{x_{n}}{y_{n-m}}, y_{n+1}=1+\frac{y_{n}}{x_{n-m}}, "$ Applied Mathematics and Letters, Vol. 17, 2004, pp. 733-737.

[4] A. Y. Ozban, "On the System of Rational Difference Equations $x_{n}=\frac{a}{y_{n-3}} \quad y_{n}=\frac{b y_{n-3}}{x_{n-q} y_{n-q}}, "$ Applied Mathematics and Computation, Vol. 188, 2007, pp. 833-837.

[5] A. S. Kurbanli, C. Cinar and I. Yalcinkaya, "On the Behavior of Positive Solutions of the System of Rational Difference Equations $x_{n}=\frac{x_{n-1}}{y_{n} x_{n-1}+1}, \quad y_{n}=\frac{y_{n-1}}{x_{n} y_{n-1}+1}, " M a-$ thematical and Computer Modelling, Vol. 53, 2011, pp. 1261-1267.

[6] G. Papaschinopoulos, "On a System of Two Nonlinear Difference Equations," Journal of Mathematicsal Analysis and Applications, Vol. 219, 1998, pp. 415-426.

[7] G. Papaschinopoulos, M. Radin and C. J. Schinas, "Study of the Asymptotic Behavior of the Solutions of Three Systems of Difference Equations of Exponential Form," Applied Mathematics and Computation, Vol. 218, 2012, pp. 5310-5318.

[8] E. Camouzis, C. M. Kent, G. Ladas and C. D. Lynd, "On the Global Character of Solutions of the System:

$x_{n+1}=\frac{\alpha_{1}+y_{n}}{x_{n}} \quad y_{n+1}=\frac{\alpha_{2}+\beta_{2} x_{n}+\gamma_{2} y_{n}}{A_{2}+B_{2} x_{n}+C_{2} y_{n}}$," Journal of Difference Equations and Applications, Vol. 18, 2012, pp. 1205-1252.

[9] E. Camouzis, E. Drymonis, G. Ladas and W. Tikjha, "Patterns of Boundedness of the Rational System

$x_{n+1}=\frac{\alpha_{1}}{A_{1}+B_{1} x_{n}+C_{1} y_{n}} \quad y_{n+1}=\frac{\alpha_{2}+\beta_{2} x_{n}+\gamma_{2} y_{n}}{A_{2}+B_{2} x_{n}+C_{2} y_{n}}, "$ Journal of Difference Equations and Applications, Vol. 18, 2012, pp. 89-110. 